\title{
On the Stability of One-Dimensional Wave Equation
}

\author{
Soon-Mo Jung \\ Mathematics Section, College of Science and Technology, Hongik University, Sejong 339-701, Republic of Korea \\ Correspondence should be addressed to Soon-Mo Jung; smjung@hongik.ac.kr \\ Received 5 August 2013; Accepted 16 September 2013 \\ Academic Editors: K. Ammari, I. Canak, and M. M. Cavalcanti \\ Copyright (C) 2013 Soon-Mo Jung. This is an open access article distributed under the Creative Commons Attribution License, which \\ permits unrestricted use, distribution, and reproduction in any medium, provided the original work is properly cited.
}

We prove the generalized Hyers-Ulam stability of the one-dimensional wave equation, $u_{t t}=c^{2} u_{x x}$, in a class of twice continuously differentiable functions.

\section{Introduction}

In 1940, Ulam [1] gave a wide ranging talk before the mathematics club of the University of Wisconsin in which he discussed a number of important unsolved problems. Among those was the question concerning the stability of group homomorphisms:

Let $G_{1}$ be a group and let $G_{2}$ be a metric group with the metric $d(\cdot, \cdot)$. Given $\varepsilon>0$, does there exist $a \delta>0$ such that if a function $h: G_{1} \rightarrow G_{2}$ satisfies the inequality $d(h(x y), h(x) h(y))<\delta$, for all $x, y \in G_{1}$, then there exists a homomorphism $H: G_{1} \rightarrow G_{2}$ with $d(h(x), H(x))<\varepsilon$, for all $x \in G_{1}$ ?

The case of approximately additive functions was solved by Hyers [2] under the assumption that $G_{1}$ and $G_{2}$ are Banach spaces. Indeed, he proved that each solution of the inequality $\|f(x+y)-f(x)-f(y)\| \leq \varepsilon$, for all $x$ and $y$, can be approximated by an exact solution, say an additive function. In this case, the Cauchy additive functional equation, $f(x+y)=$ $f(x)+f(y)$, is said to have the Hyers-Ulam stability.

Rassias [3] attempted to weaken the condition for the bound of the norm of the Cauchy difference as follows:

$$
\|f(x+y)-f(x)-f(y)\| \leq \varepsilon\left(\|x\|^{p}+\|y\|^{p}\right)
$$

and proved Hyers' theorem. That is, Rassias proved the generalized Hyers-Ulam stability (or Hyers-Ulam-Rassias stability) of the Cauchy additive functional equation. Since then, the stability of several functional equations has been extensively investigated [4-9].
The terminologies, the generalized Hyers-Ulam stability, and the Hyers-Ulam stability can also be applied to the case of other functional equations, differential equations, and various integral equations.

Given a real number $c>0$, the partial differential equation

$$
u_{t t}(x, t)-c^{2} u_{x x}(x, t)=0
$$

is called the (one-dimensional) wave equation, where $u_{t t}(x, t)$ and $u_{x x}(x, t)$ denote the second time derivative and the second space derivative of $u(x, t)$, respectively.

Let $\varphi: \mathbb{R} \times \mathbb{R} \rightarrow[0, \infty)$ be a function. If, for each twice continuously differentiable function $u: \mathbb{R} \times \mathbb{R} \rightarrow \mathbb{C}$ satisfying

$$
\left|u_{t t}(x, t)-c^{2} u_{x x}(x, t)\right| \leq \varphi(x, t) \quad(x, t \in \mathbb{R}),
$$

there exist a solution $u_{0}: \mathbb{R} \times \mathbb{R} \rightarrow \mathbb{C}$ of the (one-dimensional) wave equation (2) and a function $\Phi: \mathbb{R} \times \mathbb{R} \rightarrow[0, \infty)$ such that

$$
\left|u(x, t)-u_{0}(x, t)\right| \leq \Phi(x, t) \quad(x, t \in \mathbb{R}),
$$

where $\Phi(x, t)$ is independent of $u(x, t)$ and $u_{0}(x, t)$, then we say that the wave equation (2) has the generalized HyersUlam stability (or the Hyers-Ulam-Rassias stability).

In this paper, using an idea from [10], we prove the generalized Hyers-Ulam stability of the (one-dimensional) wave equation (2). 


\section{Generalized Hyers-Ulam Stability}

In the following theorem, using the d'Alembert method (method of characteristic coordinates), we prove the generalized Hyers-Ulam stability of the (one-dimensional) wave equation (2).

Theorem 1. Let a function $\varphi: \mathbb{R} \times \mathbb{R} \rightarrow[0, \infty)$ be given such that the double integral

$$
\int_{0}^{b} \int_{0}^{a} \varphi\left(\frac{\mu+\nu}{2}, \frac{\mu-v}{2 c}\right) d \mu d \nu
$$

exists for all $a, b \in \mathbb{R}$. If a twice continuously differentiable function $u: \mathbb{R} \times \mathbb{R} \rightarrow \mathbb{C}$ satisfies the inequality

$$
\left|u_{t t}(x, t)-c^{2} u_{x x}(x, t)\right| \leq \varphi(x, t)
$$

for all $x, t \in \mathbb{R}$, then there exists a solution $u_{0}: \mathbb{R} \times \mathbb{R} \rightarrow \mathbb{C}$ of the wave equation (2) which satisfies

$$
\begin{aligned}
& \left|u(x, t)-u_{0}(x, t)\right| \\
& \quad \leq \frac{1}{4 c^{2}}\left|\int_{0}^{x-c t} \int_{0}^{x+c t} \varphi\left(\frac{\mu+\nu}{2}, \frac{\mu-\nu}{2 c}\right) d \mu d \nu\right|
\end{aligned}
$$

for all $x, t \in \mathbb{R}$.

Proof. Let us define a function $v: \mathbb{R} \times \mathbb{R} \rightarrow \mathbb{C}$ by

$$
v(w, z):=u\left(\frac{w+z}{2}, \frac{w-z}{2 c}\right) .
$$

If we set $w=x+c t$ and $z=x-c t$, then we have $u(x, t)=$ $v(w, z)$ and

$$
\begin{aligned}
u_{t}(x, t)= & v_{w}(w, z) \frac{\partial w}{\partial t}+v_{z}(w, z) \frac{\partial z}{\partial t} \\
= & c v_{w}(w, z)-c v_{z}(w, z) \\
u_{t t}(x, t)= & c v_{w w}(w, z) \frac{\partial w}{\partial t}+c v_{w z}(w, z) \frac{\partial z}{\partial t} \\
& -c v_{z w}(w, z) \frac{\partial w}{\partial t}-c v_{z z}(w, z) \frac{\partial z}{\partial t} \\
= & c^{2} v_{w w}(w, z)-2 c^{2} v_{w z}(w, z)+c^{2} v_{z z}(w, z) \\
u_{x}(x, t)= & v_{w}(w, z) \frac{\partial w}{\partial x}+v_{z}(w, z) \frac{\partial z}{\partial x} \\
= & v_{w}(w, z)+v_{z}(w, z), \\
u_{x x}(x, t)= & v_{w w}(w, z) \frac{\partial w}{\partial x}+v_{w z}(w, z) \frac{\partial z}{\partial x} \\
& +v_{z w}(w, z) \frac{\partial w}{\partial x}+v_{z z}(w, z) \frac{\partial z}{\partial x} \\
= & v_{w w}(w, z)+2 v_{w z}(w, z)+v_{z z}(w, z)
\end{aligned}
$$

for all $x, t \in \mathbb{R}$. Hence, we have

$$
u_{t t}(x, t)-c^{2} u_{x x}(x, t)=-4 c^{2} v_{w z}(w, z),
$$

for any $x, t \in \mathbb{R}$. Thus, it follows from inequality (6) that

$$
\left|v_{w z}(w, z)\right| \leq \frac{1}{4 c^{2}} \varphi\left(\frac{w+z}{2}, \frac{w-z}{2 c}\right)
$$

for any $w, z \in \mathbb{R}$.

Therefore, we get

$$
\begin{aligned}
-\frac{1}{4 c^{2}} & \int_{0}^{z} \int_{0}^{w} \varphi\left(\frac{\mu+\nu}{2}, \frac{\mu-\nu}{2 c}\right) d \mu d \nu \\
& \leq \int_{0}^{z} \int_{0}^{w} v_{w z}(\mu, \nu) d \mu d \nu \\
& \leq \frac{1}{4 c^{2}} \int_{0}^{z} \int_{0}^{w} \varphi\left(\frac{\mu+\nu}{2}, \frac{\mu-\nu}{2 c}\right) d \mu d \nu
\end{aligned}
$$

or equivalently

$$
\begin{aligned}
& |v(w, z)-v(w, 0)-v(0, z)+v(0,0)| \\
& \quad \leq \frac{1}{4 c^{2}}\left|\int_{0}^{z} \int_{0}^{w} \varphi\left(\frac{\mu+\nu}{2}, \frac{\mu-\nu}{2 c}\right) d \mu d \nu\right|,
\end{aligned}
$$

for all $w, z \in \mathbb{R}$.

On account of (8), we get

$$
\begin{array}{cl}
v(w, z)=u\left(\frac{w+z}{2}, \frac{w-z}{2 c}\right), & v(w, 0)=u\left(\frac{w}{2}, \frac{w}{2 c}\right) \\
v(0, z)=u\left(\frac{z}{2},-\frac{z}{2 c}\right), & v(0,0)=u(0,0) .
\end{array}
$$

Hence, it follows from (13) and the last equalities that

$$
\begin{aligned}
& \left|u\left(\frac{w+z}{2}, \frac{w-z}{2 c}\right)-u\left(\frac{w}{2}, \frac{w}{2 c}\right)-u\left(\frac{z}{2},-\frac{z}{2 c}\right)+u(0,0)\right| \\
& \leq \frac{1}{4 c^{2}}\left|\int_{0}^{z} \int_{0}^{w} \varphi\left(\frac{\mu+\nu}{2}, \frac{\mu-v}{2 c}\right) d \mu d \nu\right|
\end{aligned}
$$

for all $w, z \in \mathbb{R}$.

If we set $w=x+c t$ and $z=x-c t$ in the last inequality, then we obtain

$$
\begin{aligned}
& \left|u(x, t)-u_{0}(x, t)\right| \\
& \quad \leq \frac{1}{4 c^{2}}\left|\int_{0}^{x-c t} \int_{0}^{x+c t} \varphi\left(\frac{\mu+\nu}{2}, \frac{\mu-\nu}{2 c}\right) d \mu d \nu\right|,
\end{aligned}
$$

for all $x, t \in \mathbb{R}$, where we set

$$
\begin{aligned}
u_{0}(x, t):= & u\left(\frac{x}{2}+\frac{c}{2} t, \frac{x}{2 c}+\frac{t}{2}\right) \\
& +u\left(\frac{x}{2}-\frac{c}{2} t,-\frac{x}{2 c}+\frac{t}{2}\right)-u(0,0) .
\end{aligned}
$$


By some tedious calculations, we get

$$
\begin{aligned}
& \frac{\partial}{\partial t} u_{0}(x, t) \\
& =\frac{c}{2} u_{x}\left(\frac{x}{2}+\frac{c t}{2}, \frac{x}{2 c}+\frac{t}{2}\right)+\frac{1}{2} u_{t}\left(\frac{x}{2}+\frac{c t}{2}, \frac{x}{2 c}+\frac{t}{2}\right) \\
& -\frac{c}{2} u_{x}\left(\frac{x}{2}-\frac{c t}{2},-\frac{x}{2 c}+\frac{t}{2}\right)+\frac{1}{2} u_{t}\left(\frac{x}{2}-\frac{c t}{2},-\frac{x}{2 c}+\frac{t}{2}\right), \\
& \frac{\partial^{2}}{\partial t^{2}} u_{0}(x, t) \\
& =\frac{c^{2}}{4} u_{x x}\left(\frac{x}{2}+\frac{c t}{2}, \frac{x}{2 c}+\frac{t}{2}\right)+\frac{c}{2} u_{x t}\left(\frac{x}{2}+\frac{c t}{2}, \frac{x}{2 c}+\frac{t}{2}\right) \\
& +\frac{1}{4} u_{t t}\left(\frac{x}{2}+\frac{c t}{2}, \frac{x}{2 c}+\frac{t}{2}\right)+\frac{c^{2}}{4} u_{x x}\left(\frac{x}{2}-\frac{c t}{2},-\frac{x}{2 c}+\frac{t}{2}\right) \\
& -\frac{c}{2} u_{x t}\left(\frac{x}{2}-\frac{c t}{2},-\frac{x}{2 c}+\frac{t}{2}\right)+\frac{1}{4} u_{t t}\left(\frac{x}{2}-\frac{c t}{2},-\frac{x}{2 c}+\frac{t}{2}\right), \\
& \frac{\partial}{\partial x} u_{0}(x, t) \\
& =\frac{1}{2} u_{x}\left(\frac{x}{2}+\frac{c t}{2}, \frac{x}{2 c}+\frac{t}{2}\right)+\frac{1}{2 c} u_{t}\left(\frac{x}{2}+\frac{c t}{2}, \frac{x}{2 c}+\frac{t}{2}\right) \\
& +\frac{1}{2} u_{x}\left(\frac{x}{2}-\frac{c t}{2},-\frac{x}{2 c}+\frac{t}{2}\right)-\frac{1}{2 c} u_{t}\left(\frac{x}{2}-\frac{c t}{2},-\frac{x}{2 c}+\frac{t}{2}\right) \text {, } \\
& \frac{\partial^{2}}{\partial x^{2}} u_{0}(x, t) \\
& =\frac{1}{4} u_{x x}\left(\frac{x}{2}+\frac{c t}{2}, \frac{x}{2 c}+\frac{t}{2}\right)+\frac{1}{2 c} u_{x t}\left(\frac{x}{2}+\frac{c t}{2}, \frac{x}{2 c}+\frac{t}{2}\right) \\
& +\frac{1}{4 c^{2}} u_{t t}\left(\frac{x}{2}+\frac{c t}{2}, \frac{x}{2 c}+\frac{t}{2}\right)+\frac{1}{4} u_{x x}\left(\frac{x}{2}-\frac{c t}{2},-\frac{x}{2 c}+\frac{t}{2}\right) \\
& -\frac{1}{2 c} u_{x t}\left(\frac{x}{2}-\frac{c t}{2},-\frac{x}{2 c}+\frac{t}{2}\right)+\frac{1}{4 c^{2}} u_{t t}\left(\frac{x}{2}-\frac{c t}{2},-\frac{x}{2 c}+\frac{t}{2}\right) \text {, }
\end{aligned}
$$

for all $x, t \in \mathbb{R}$. Hence, we know that

$$
\frac{\partial^{2}}{\partial t^{2}} u_{0}(x, t)-c^{2} \frac{\partial^{2}}{\partial x^{2}} u_{0}(x, t)=0,
$$

for any $x, t \in \mathbb{R}$; that is, $u_{0}(x, t)$ is a solution of the wave equation (2).

Corollary 2. Given a constant $\alpha>0$, let a function $\varphi: \mathbb{R} \times$ $\mathbb{R} \rightarrow[0, \infty)$ be given as

$$
\varphi(x, t)=\alpha e^{-x^{2}-c^{2} t^{2}} .
$$

If a twice continuously differentiable function $u: \mathbb{R} \times \mathbb{R} \rightarrow \mathbb{C}$ satisfies inequality (6), for all $x, t \in \mathbb{R}$, then there exists a solution $u_{0}: \mathbb{R} \times \mathbb{R} \rightarrow \mathbb{C}$ of the wave equation (2) which satisfies

$$
\left|u(x, t)-u_{0}(x, t)\right| \leq \frac{\alpha \pi}{8 c^{2}}\left|\operatorname{erf}\left(\frac{x-c t}{\sqrt{2}}\right) \operatorname{erf}\left(\frac{x+c t}{\sqrt{2}}\right)\right|,
$$

for all $x, t \in \mathbb{R}$.
Proof. Since

$$
\begin{aligned}
& \left|\int_{0}^{b} \int_{0}^{a} \varphi\left(\frac{\mu+\nu}{2}, \frac{\mu-\nu}{2 c}\right) d \mu d \nu\right| \\
& \quad=\left|\int_{0}^{b} \int_{0}^{a} \alpha e^{-\mu^{2} / 2-\nu^{2} / 2} d \mu d \nu\right| \\
& \quad=\alpha\left|\int_{0}^{b} e^{-\nu^{2} / 2} d \nu\right|\left|\int_{0}^{a} e^{-\mu^{2} / 2} d \mu\right| \\
& \quad=2 \alpha \frac{\pi}{4}\left|\left(\frac{2}{\sqrt{\pi}} \int_{0}^{b / \sqrt{2}} e^{-\nu^{2}} d \nu\right)\left(\frac{2}{\sqrt{\pi}} \int_{0}^{a / \sqrt{2}} e^{-\mu^{2}} d \mu\right)\right| \\
& \quad=\frac{\alpha \pi}{2}\left|\operatorname{erf}\left(\frac{b}{\sqrt{2}}\right) \operatorname{erf}\left(\frac{a}{\sqrt{2}}\right)\right|<\infty,
\end{aligned}
$$

for all $a, b \in \mathbb{R}$, in view of Theorem 1 , we conclude that the statement of this corollary is true.

\section{Conflict of Interests}

The author declares that there is no conflict of interests regarding the publication of this paper.

\section{Acknowledgments}

This research was supported by the Basic Science Research Program through the National Research Foundation of Korea (NRF) funded by the Ministry of Education (no. 2013R1A1A2005557).

\section{References}

[1] S. M. Ulam, A Collection of Mathematical Problems, Interscience, New York, NY, USA, 1960.

[2] D. H. Hyers, "On the stability of the linear functional equation," Proceedings of the National Academy of Sciences of USA, vol. 27, pp. 222-224, 1941.

[3] T. M. Rassias, "On the stability of the linear mapping in Banach spaces," Proceedings of the American Mathematical Society, vol. 72, pp. 297-300, 1978.

[4] G. L. Forti, "Hyers-Ulam stability of functional equations in several variables," Aequationes Mathematicae, vol. 50, no. 1-2, pp. 143-190, 1995.

[5] P. Găvrută, "A generalization of the Hyers-Ulam-Rassias stability of approximately additive mappings," Journal of Mathematical Analysis and Applications, vol. 184, no. 3, pp. 431-436, 1994.

[6] D. H. Hyers, G. Isac, and T. M. Rassias, Stability of Functional Equations of Several Variables, Birkhauser, Boston, Mass, USA, 1998.

[7] D. H. Hyers and T. M. Rassias, "Approximate homomorphisms," Aequationes Mathematicae, vol. 44, no. 2-3, pp. 125-153, 1992.

[8] S.-M. Jung, Hyers-Ulam-Rassias Stability of Functional Equations in Nonlinear Analysis, vol. 48 of Springer Optimization and Its Applications, Springer, New York, NY, USA, 2011.

[9] T. M. Rassias, "On the stability of functional equations and a problem of Ulam," Acta Applicandae Mathematicae, vol. 62, no. 1, pp. 23-130, 2000.

[10] B. Hegyi and S.-M. Jung, "On the stability of Laplace's equation," Applied Mathematics Letters, vol. 26, pp. 549-552, 2013. 


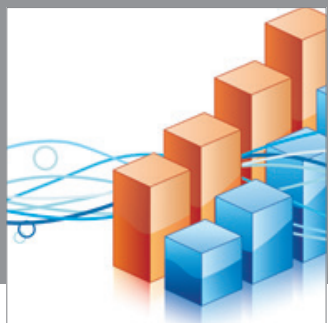

Advances in

Operations Research

mansans

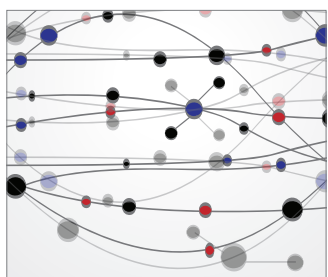

The Scientific World Journal
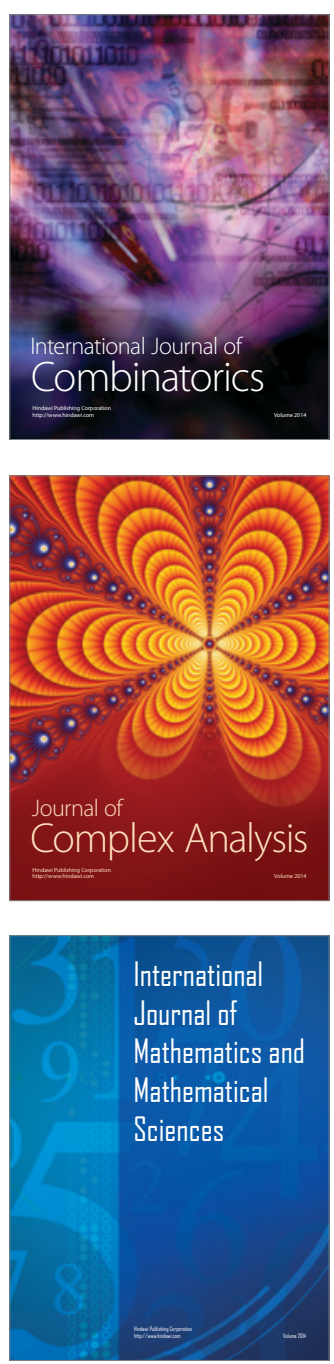
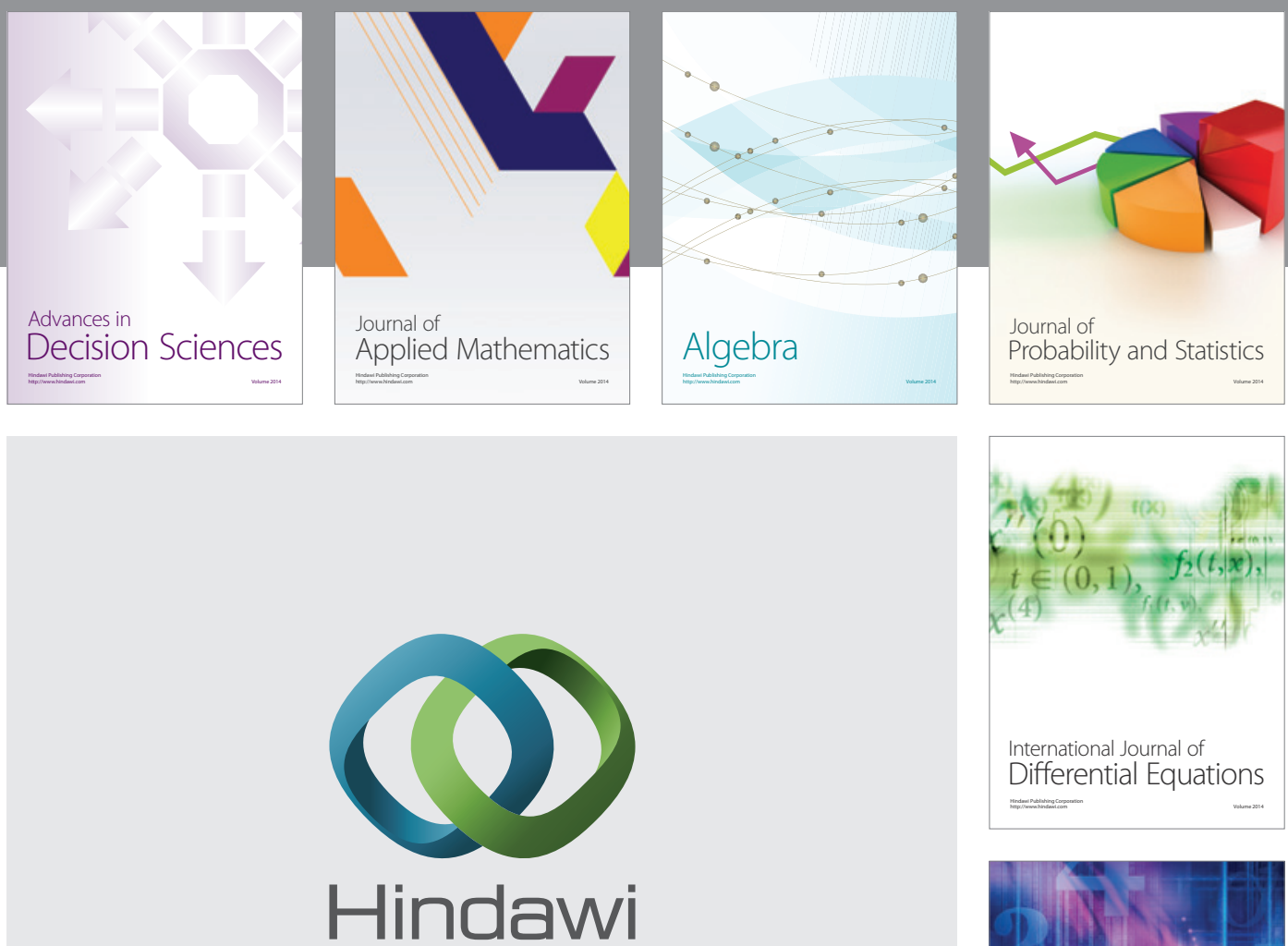

Submit your manuscripts at http://www.hindawi.com
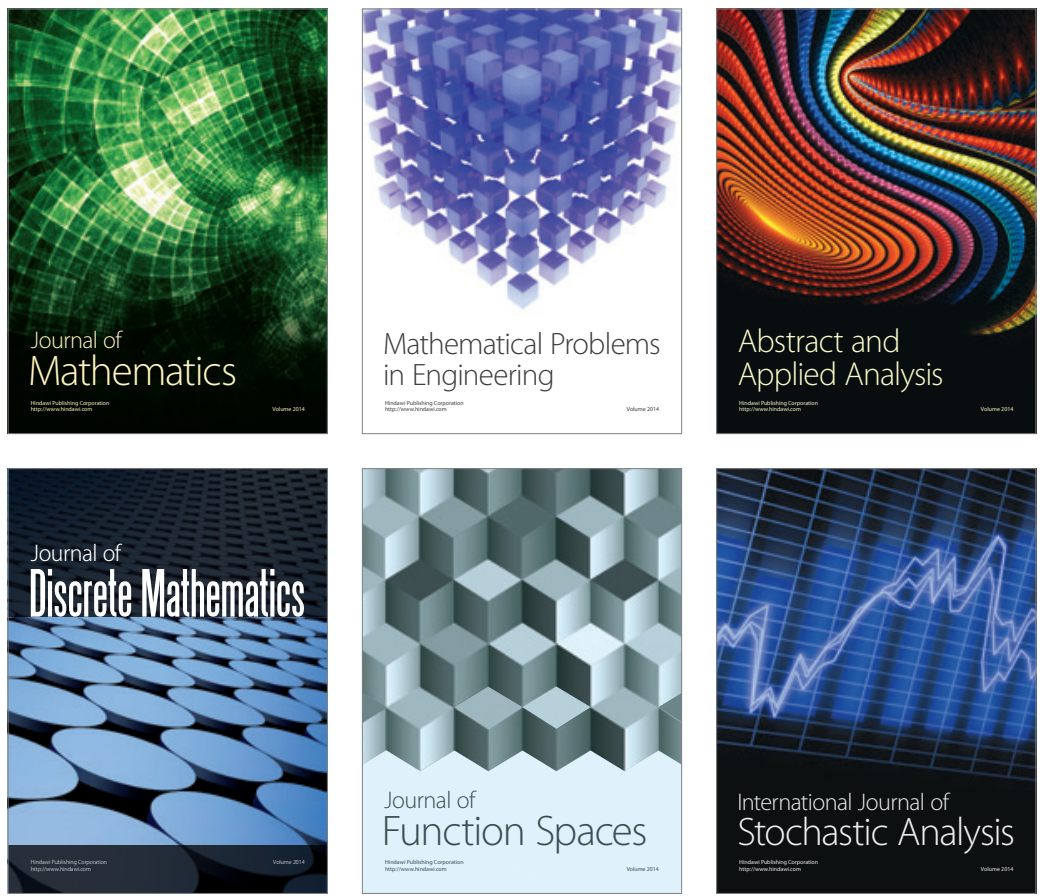

Journal of

Function Spaces

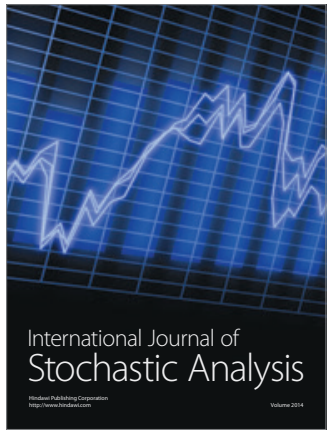

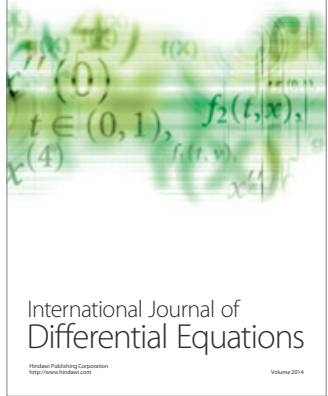
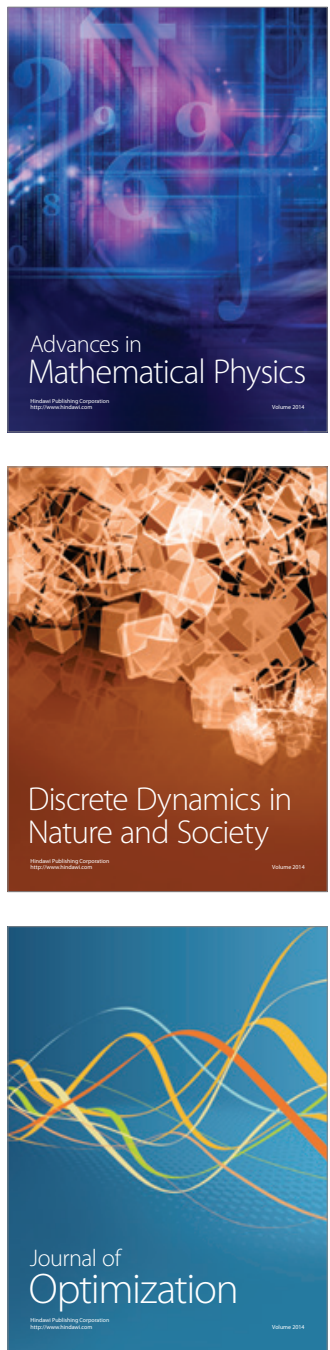